\title{
A Missing Step: The Value of Psychological Mindedness Training during Psychiatry Residency
}

\author{
Mark Cogburn', Nahrain Raihan1, Hannah Scott², Hannah Cogburn ${ }^{3}$ \\ ${ }^{1}$ LSU Health-Shreveport; Louisiana State University, Baton Rouge, LA, USA \\ ${ }^{2}$ LSU Health Sciences Center-New Orleans; Louisiana State University, Baton Rouge, LA, USA \\ ${ }^{3}$ University of North Carolina; Chapel Hill, NC, USA \\ Email: mark.cogburn@lsuhs.edu,nraihana443@gmail.com, Hscot1@lsuhsc.edu,hkc711@email.unc.edu
}

How to cite this paper: Cogburn, M., Raihan, N., Scott, H. and Cogburn, H. (2022) A Missing Step: The Value of Psychological Mindedness Training during Psychiatry Residency. Open Journal of Psychiatry, 12, 73-77.

https://doi.org/10.4236/ojpsych.2022.121007

Received: November 3, 2021

Accepted: January 8, 2022

Published: January 11, 2022

Copyright $\odot 2022$ by author(s) and Scientific Research Publishing Inc. This work is licensed under the Creative Commons Attribution International License (CC BY 4.0). http://creativecommons.org/licenses/by/4.0/

\section{Open Access}

\begin{abstract}
Psychiatry residents, who lack psychological mindedness, struggle to understand the multiple determinants of behavior, or the numerous meanings of a single symptom. This is unfortunate because most psychiatry resident interns feel that they are finally among the group where they belong... like-minded physicians who share a fascination with human nature and a desire to grasp all the nuances and complexities of human behavior. This paper provides a framework for psychiatry departments to train residents along a continuum of psychological mindedness. The authors explain the concept of psychological mindedness, explore the pros and cons of acquiring psychological mindedness, and a scientific basis for its use in general psychiatry. A new model is included to capture any resident's ability to become psychologically minded, with direction for Program Director's to possibly develop growth in a resident's psychological mindedness over four years of training.
\end{abstract}

\section{Keywords}

Psychological Mindedness, Psychiatry, Residents, House Officers, Psychotherapy, Training

\section{Literature Review}

Psychological Mindedness (PM) is the tendency to look for motives, patterns, or distortions in oneself and others and is likely a trait that consists of an effective and intellectual component that is prized and developed for themselves and their patients [1]. In this sense, PM, when focused inward, is a particular form of introspection or self-awareness that is informed by knowledge (not necessarily 
academic, sophisticated, or technical) of psychological processes. Whilst several studies have illustrated the importance of empathy, trust, and the better understanding of one's patient as a keystone in building a good patient-physician relationship, studies describe the deterioration of empathy during medical school, specifically in the clinical $3^{\text {rd }}$ year, which ironically is when empathy is required the most [2].

Ideally, psychiatry residents should have an underlying ability to see the relationships between feelings, thoughts and behaviors, with the goal to learn the meanings and causes of experiences and behavior. Psychological mindedness helps to develop empathy and altruism. The authors believe that after a patient's psychological processes are observed by a resident, a highly psychologically minded resident is in a position to hypothesize about the origin, maintenance strategies, and functions of the patient's behaviors, thoughts, and feelings. It is also the author's belief that without empathy, the low PM residents' hypotheses will be simply guessing at best. Residents with high PM should tolerate ambiguity, while low PM residents likely struggle as they search for concrete explanations of behavior.

Physician empathy, in an outpatient clinical setting, is associated with patients managing their chronic illnesses effectively. After following 891 patients with Diabetes Mellitus and differentiating each physician, using the Jefferson Scale of Empathy, into "low, moderate, and high empathy," it was noted that those patients who were treated by "high empathy" physicians showed good control of hemoglobin Alc (56\%) versus those who were treated by "low empathy" physicians $(40 \%, \mathrm{p}<0.001)$. In addition, patients with "high empathy" doctors showed better control of LDL-C (59\%) compared to those patients with "low empathy" doctors $(44 \%, \mathrm{p}<0.001)[3]$.

The effects of empathy, displayed in the form of physician-patient communication, using verbal and nonverbal cues, and the quality of the encounter can lead to patient satisfaction and better compliance. Mutual understanding and trust between the patient and doctor strengthened, thus resulting in better meeting the patient's needs, the patient feeling heard, understood, and helped, and creating a sense of patient empowerment and control over his or her illness [3].

Further evidence of improved prognosis was seen in the study by Rakel [4], where patients with the common cold, who perceived their physicians as empathetic and understanding, had a shorter and less impactful course of illness. Those that believed their doctors took the time to understand them and their unique situation felt more inclined to divulge information, that ultimately assisted in formulating an accurate diagnosis, treatment plan, and leading to improved compliance and prognosis.

Although there are many factors that improve patient compliance, a clinician attempting to understand the individual and their past experiences leads to trust, feeling understood, and genuinely cared for. This in turn, promotes cooperation and a successful alliance between psychiatrist and patient. Treatment compliance 
was found to be "nearly three times higher" in primary care relationships that showed high levels of trust and seeing the patient as an individual rather than a set of symptoms [5].

Finally, there is evidence that inviting low PM persons into groups with high PM others can be an effective tool in increasing PM. Psychological mindedness is inversely related to personal distress, indicating an ability to remain calm and attentive during emergency situations [6]. Based upon Piper's research, program directors may want to consider blending Low PM residents with high PM residents to process patient's wishes, fears, defenses, and relationship to the patients presenting problems [7] and that this process is enhanced during group psychotherapy training as high PM persons reveal methods to gain insight and other's psychological states [8].

\section{Method}

Each Psychiatry resident should initially complete the 21 items studied by Shill and Lumley [9] from the Psychological Mindedness Scale [10]. A suggestion for convenience and brevity is to simplify these items into a true/false format where each question represents one point. For residents who score below 10, the supervisor should consider having the resident complete the Psychological Perspective Taking and Empathic Concern Scales on the Interpersonal Reactivity Index [11]. Again, transcribing these questions to true/false items instead of Likert scaling is preferred. Supervisors are encouraged to give their attention to those who score above an eight which indicates some degree of psychological mindedness. For continuity, consideration to repeating this screening annually over the course of the four years of training allows for resident feedback pursuant to the PM development.

The intended goal of this intervention is to acknowledge that all scores, low and high, are helpful in regard to training psychiatric residents. The authors suggest that supervisors are to avoid any criticism of a resident based upon PM score, but use them in a broader effort that acknowledges "a hat for every head". This approach allows PM training to be comfortable for each resident. This model is helpful to the types of psychotherapy intern supervisors may want to consider. Those who initially score lower in PM, should receive early exposure to less ambiguous interventions in their first year of training e.g. behavior modification, problem-solving therapy, assertiveness training, psychopharmacology, neurology, and genetics. High PM residents can also be introduced to person-centered therapy, existentialism, family of origin concepts, psychodynamic and gestalt techniques. By considering baseline scores as a "starting point" for $\mathrm{PM}$, instead of a fixed trait, this screening may diminish any pejorative notion of a "lesser than" and "elite group".

\section{Conclusions and Recommendations}

Understanding the importance of incorporating psychological mindedness when 
treating patients yet observing the lack of such training in current medical curriculum, creates a need to establish and implement a program that would methodically train medical professionals based on their baseline skill set to better serve their patients.

It is the goal of the authors to avoid any criticism of a resident based upon PM score, but to acknowledge "a hat for every head" and begin PM training where the resident initially feels more comfortable. One possible limitation to this approach evolves around Farber's literature review on the cons, or disadvantages, of psychological mindedness [12]. He noted that highly psychologically minded people often had an inhibition to spontaneity and action, self-doubt, guilt, emotional detachment, distress anxiety, depression, and somatic symptoms. His general conclusion was that those with "high psychological mindedness" were more unwilling to share their emotions.

A ubiquitous, but tolerable, risk of PM training is the early application of the concepts to everyone, resulting in a trainee discounting honest and simple patient behavior as a complex display of internal motives and drives. There is an additional issue of residents constantly interpreting each other's behaviors, which may not be appreciated by other house officers or simply become tiresome. A third caveat may yield PM inspired residents feel cheated by superficial directives from their administrators about duties and assignments. Moreover, while, individuals with high PM are thought to be more insightful [13] and emotionally aware [11], individuals may become more interpersonally distant [13] and self-critical [14] than those who are not psychologically minded. Perhaps this could be conducted as part of weekly case conferences.

It is understood that reflection is a necessary precondition to insight [15]. Moreover, some psychiatry residents have an interest in psychological issues yet engage in limited self-reflection resulting in little gain in insight about themselves or others. Teaching psychological mindedness is a means to enhance both of these prerequisite aspects of psychiatry. Focusing on these hallmarks of good psychiatry should be considered as a part of weekly case conferences with supervisors.

\section{Author Contributions}

Conceptualization, MC, NR, HS; Methodology, MC, NR; Resources, MC, NR; Writing-original draft preparation, MC, NR; Writing-review and editing, MC, NR, HC. All authors have read and agreed to the published version of the manuscript.

\section{Funding}

This work received no external funding.

\section{Conflicts of Interest}

The authors declare no conflict of interest. 


\section{References}

[1] Farber, B.A. (1985) The Genesis, Development, and Implications of Psychological-Mindedness in Psychotherapists. Psychotherapy. Theory, Research, Practice, Training, 22, 170-177. https://doi.org/10.1037/h0085490

[2] Norfolk, T., Birdi, K. and Walsh, D. (2007) The Role of Empathy in Establishing Rapport in the Consultation: A New Model. Medical Education, 41, 690-697. https://doi.org/10.1111/j.1365-2923.2007.02789.x

[3] Hojat, M., Louis, D.Z., Markham, F.W., Wender, R., Rabinowitz, C. and Gonnella, J.S. (2011) Physicians' Empathy and Clinical Outcomes for Diabetic Patients. Academic Medicine, 86, 359-364. https://doi.org/10.1097/ACM.0b013e3182086fe1

[4] Rakel, D.P., Hoeft, T.J., Barrett, B.P., Chewning, B.A., Craig, B.M. and Niu, M. (2009) Practitioner Empathy and the Duration of the Common Cold. Family Medicine, 41, 494-501.

[5] Martin, L.R., Williams, S.L., Haskard, K.B. and Dimatteo, M.R. (2005) The Challenge of Patient Adherence. Therapeutics and Clinical Risk Management, 1, 189-199.

[6] Piper, W.E., Joyce, A.S., Rosie, J.S. and Azim, H.F. (1994) Psychological Mindedness, Work, and Outcome in Day Treatment. International Journal of Group Psychotherapy, 44, 291-311. https://doi.org/10.1080/00207284.1994.11490755

[7] Rai, S., Punia, V., Choudhury, S. and Mathew, K.J. (2015) Psychological Mindedness: An Overview. Indian Journal of Positive Psychology, 6, 127-132.

[8] Björgvinsson, T. and Hart, J. (2006) Cognitive Behavioral Therapy Promotes Mentalizing. In: Allen, J.G. and Fonagy, P., Eds., Handbook of Mentalization-Based Treatment, John Wiley \& Sons Inc., Hoboken, 157-170.

https://doi.org/10.1002/9780470712986.ch7

[9] Shill, M.A. and Lumley, M.A. (2002) The Psychological Mindedness Scale: Factor Structure, Convergent Validity and Gender in a Non-Psychiatric Sample. Psychology and Psychotherapy: Theory, Research and Practice, 75, 131-150. https://doi.org/10.1348/147608302169607

[10] Conte, H.R., Ratto, R. and Karasu, T.B. (1996) The Psychological Mindedness Scale: Factor Structure and Relationship to Outcome of Psychotherapy. The Journal of Psychotherapy Practice and Research, 5, 250-259.

[11] Davis, M.H. (1983) Measuring Individual Differences in Empathy: Evidence for a Multidimensional Approach. Journal of Personality and Social Psychology, 44, 113-126. https://doi.org/10.1037/0022-3514.44.1.113

[12] Farber, B.A. (1989) Psychological-Mindedness: Can There Be Too Much of a Good Thing? Psychotherapy: Theory, Research, Practice, Training, 26, 210-217. https://doi.org/10.1037/h0085421

[13] Appelbaum, S.A. (1973) Psychological-Mindedness: Word, Concept and Essence. The International Journal of Psychoanalysis, 54, 35-46.

[14] Henry, W., Sims, J. and Spray, S.L. (1973) Public and Private Lives of Psychotherapists. Jossey-Bass, San Francisco.

[15] Grant, A.M. (2001) Rethinking Psychological Mindedness: Metacognition, SelfReflection, and Insight. Behaviour Change, 18, 8-17.

https://doi.org/10.1375/bech.18.1.8 